Ann. Zootech., I977, 26 (4), 513-522.

\title{
Utilisation des tourteaux traités au formol par les vaches laitières.
}

\section{III. - Influence sur l'aminoacidémie libre de la formolation des tourteaux, du niveau d'apport azoté et de la nature de l'azote alimentaire}

\author{
C. CHAMPREDON, R. VÉRITÉ (*), J. PRUGNAUD et R. PION
}

\author{
Laboratoive d'Étude du Métabolisme Azoté \\ (*) Laboratoire de la Production Laitière. \\ Centve de Recherches de Clermont-Ferrand I.N.R.A. \\ Theix, Saint-Genès-Champanelle \\ 63110 Beaumont (France)
}

\begin{abstract}
Résumé
Au cours de 3 expériences réalisées en début ( $n^{\circ}$ I et II) et en milieu de lactation (no III), des vaches laitières ont été alimentées avec des rations isoénergétiques et isoazotées à base d'ensilage de maïs comportant un aliment concentré contenant des tourteaux de soja et de colza. Les effets sur les teneurs en acides aminés libres du sang du traitement des tourteaux par le formol, de la substitution des tourteaux par de l'urée et de l'abaissement du taux azoté de la ration, ont été étudiés.

L'incorporation à l'aliment concentré des tourteaux formolés a provoqué une augmentation des teneurs sanguines en valine, isoleucine, leucine, histidine et proline et une diminution de celles en glutamine ( + asparagine) ainsi que dans certains cas de celles en lysine (tabl. I, 2 et 3). Les concentrations dans le sang de la phénylalanine et surtout de la méthionine étaient toujours très faibles durant les périodes expérimentales. L'élévation des teneurs en acides aminés à chaînes ramifiées et en histidine libtes du sang sous l'effet de la formolation des tourteaux ingérés est la résultante de deux phénomènes : l'augmentation des quantités d'acides aminés qui disparaissent dans l'intestin (VífiTí: et al., I977) et l'amélioration de la production laitière des vaches (VÉRIT' et JoURNE'T, I977); l'utilisation par la glande mammaire de ces composés est ıéduite par la fourniture insuffisante de phénylalanine, lysine et surtout de méthionine aux tissus des animaux.

La distribution d'urée, de même que celle d'une ration de faible taux azoté, provoquent une diminution des teneurs sanguines de la plupart des acides aminés indispensables. L'ensemble de ces résultats permet de supposer que la méthionine et la phénylalanine sont les premiers limitants chez des vaches laitières recevant une ration à base d'ensilage de maîs.
\end{abstract}




\section{Introduction}

Le traitement par le formol d'un mélange de tourteaux de soja et de colza a permis d'augmenter la quantité de matières azotées digérées dans l'intestin (VÉRITÉ et al., I977) et d'améliorer dans 3 essais la production laitière des vaches en début ou en milieu de lactation (VÉRITÉ et Journét, I977). Au cours de ces expériences, nous avons tenté de relier les performances des vaches au taux de satisfaction de leurs besoins en acides aminés. Pour cela, nous avons étudié les variations de l'aminoacidémie libre en fonction de la source d'azote utilisée (tourteaux non traités, tourteaux formolés, urée, herbe), du niveau des apports azotés en début de lactation et du stade de lactation.

\section{Matériel et méthodes}

'Trois expériences ont été réalisées en début de lactation ( ${ }^{\circ}$ I et II) et en milieu de lactation ( $n^{\circ}$ III). Quatre-vingt-deux vaches ont été réparties en 8 lots et ont reçu une ration à base d'ensilage de maïs enrichi (expériences I et II) ou non (expérience III) en urée. Les rations étaient isoénergétiques et isoazotées sauf pour un lot d'animaux dans l'expérience II qui était fortement sous alimentée en azote. Dans chacune des expériences, un lot d'animaux recevait des tourteaux non traités et un autre des tourteaux formolés. Dans l'expérience III, les vaches d'un $3^{\mathrm{e}}$ lot recevaient un mélange de maïs grain et d'urée en remplacement des tourteaux. Après Io à $\mathbf{I} 3$ semaines d'expériences, les vaches allaient au pâturage. Les protocoles expérimentaux ainsi que les caractéristiques des rations distribuées et le traitement des tourteaux sont décrits par ailleurs (VÉRITÉ et JoURNET, I977).

Du sang était prélevé à plusieurs reprises dans la veine jugulaire de chaque vache 2 h 30 après la distribution du repas du matin en cours d'expérience et à la fin de la période post-expérimentale après la mise à 1'herbe (tabl. I, 2 et 3 ). Un échantillon moyen était réalisé avec le sang des animaux d'un même lot prélevé au même stade de chaque expérience. Le sang était recueilli dans un flacon contenant de $1^{\prime}$ 'éthanol à $95^{\circ}$ froid additionné de $2 \mathrm{p}$. cent de thiodiglycol à raison d'un volume de sang pour 7 volumes d'alcool. Les acides aminés libres étaient extraits par l'éthanol aqueux $\left(82^{\circ}\right)$ froid par épuisements successifs et dosés par chromatographie sur résine échangeuse de cations.

\section{Résultats}

Les résultats zootechniques et un certain nombre de paramètres digestifs et sanguins sont décrits et discutés dans une autre publication (VÉRITÉ et JourNET, I977).

Les teneurs en acides aminés libres du sang des vaches figurent respectivement pour chacune des 3 expériences dans les tableaux I, 2 et 3 . Le tryptophane et la cystine, non extraits quantitativement n'ont pas été dosés; la N-méthyl-1ysine n'a pas été séparée de la lysine ainsi que la glutamine de l'asparagine. 
Influence du traitement des tourteaux par le formol.

\section{- Expérience $I$.}

Les teneurs en valine, isoleucine, leucine, histidine et proline sont plus élevées dans le sang des vaches recevant les tourteaux formolés que chez celles qui consomment les tourteaux non traités. C'est l'inverse qui est observé en ce qui concerne la glutamine ( + asparagine). Les différences de concentrations sont plus importantes en $5^{\mathrm{e}} \mathrm{qu}^{\prime}$ en $\mathrm{IO}^{\mathrm{e}}$ semaine de lactation; les écarts sont plus accentués si l'on ne considère que les 8 animaux de chaque lot les plus forts producteurs (plus de $25 \mathrm{~kg}$ de lait par jour) au lieu des $\mathrm{I} 2$ vaches de chaque lot.

\section{TABLEAU I}

Aminoacidémie libre des vaches laitières (Expérience I)

Free aminoacids in blood of dairy cows (Trial I)

(mg p. cent g. de sang)

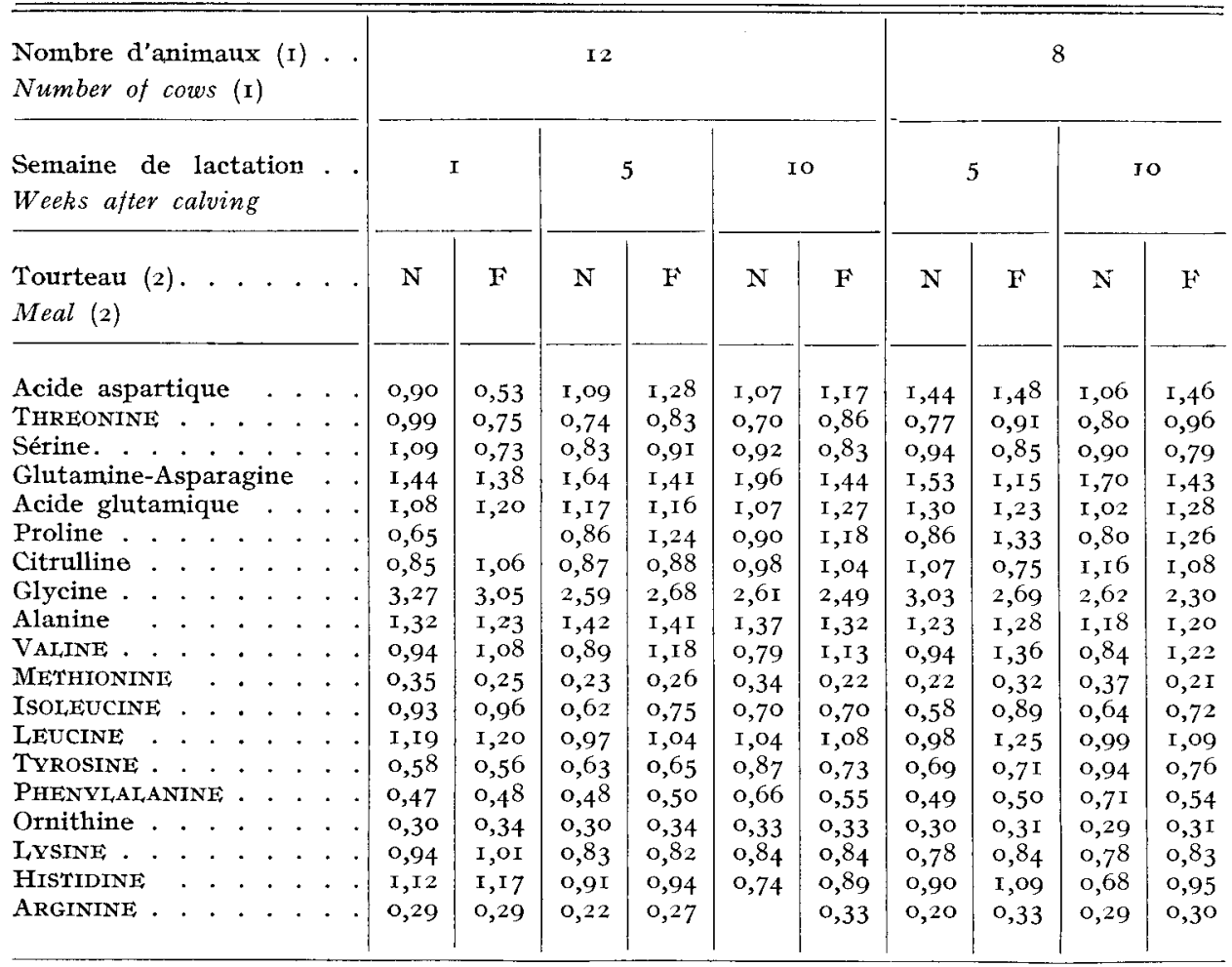

(I) 12 : ensemble des vaches de chaque lot.

8 : vaches produisant plus de $25 \mathrm{~kg}$ de lait par jour.

I2 : all cows of each group.

8 : cons producing more than $25 \mathrm{~kg}$ of milk per day.

(2) N : tourteaux non traités $\mathrm{F}$ : tourteaux formolés.

$\mathrm{N}$ : untreated meal $\mathrm{F}$ : treated meal. 


\section{-. Expérience $I I$.}

Le traitement des tourteaux par le formol se traduit par une élévation des teneurs sanguines en acides aminés à chaîne ramifiée ainsi qu'en proline et histidine, et une diminution des concentrations en tyrosine, lysine et à un moindre degré en thréonine et en glutamine ( + asparagine). Les écarts mis en évidence sont beaucoup plus importants en $2^{\mathrm{e}}$ qu'en $5^{\mathrm{e}}$ semaine de lactation.

\section{TABLEAU 2}

Aminoacidemie libre des vaches laitières (Expérience II)

Free aminoacids in blood of dairy cows (Trial II)

(mg p. cent g de sang)

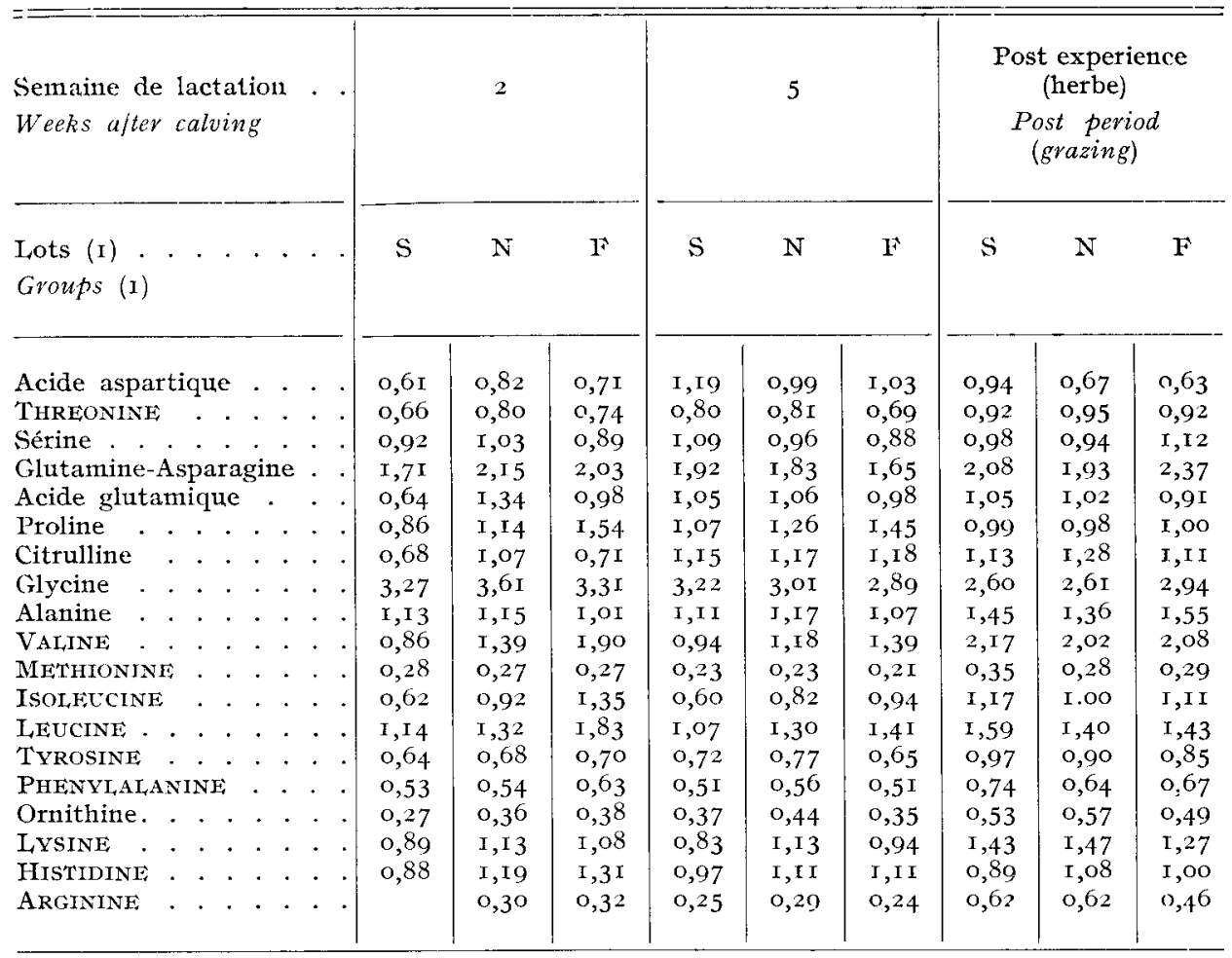

(I) S: faible teneur en azote - Low nitrogen.

$\mathrm{N}$ : tourteaux non traités - untreated meal.

$\mathrm{F}$ : tourteaux formolés - treated meal.

\section{- Expérience III.}

Les teneurs sanguines en thréonine, proline, isolencine, phénylalanine et histidine sont en général faiblement augmentées par la forniolation des tourteaux. Les différences de concentrations en acides aminés libres dans le sang des animaux recevant les 2 types de tourteaux sont peu importantes en $6 \mathrm{e}$ semaine d'expérience, 
sauf en ce qui concerne la lysine dont la valeur la plus faible est observée chez les vaches consommant les tourteaux formolés. Il n'en est pas de même au cours de la $\mathrm{I} 3^{\mathrm{e}}$ semaine : la concentration sanguine en lysine est dans ce cas légèrement plus élevée chez les vaches consommant les tourteaux formolés que chez celles recevant les tourteaux non traités.

\section{TABLEAU 3}

Aminoacidémie libre des vaches laitières (Expérience $I I I$ )

Free aminoacids in blood of dairy cows (Trial III)

(mg p. cent $\mathrm{g}$ de sang)

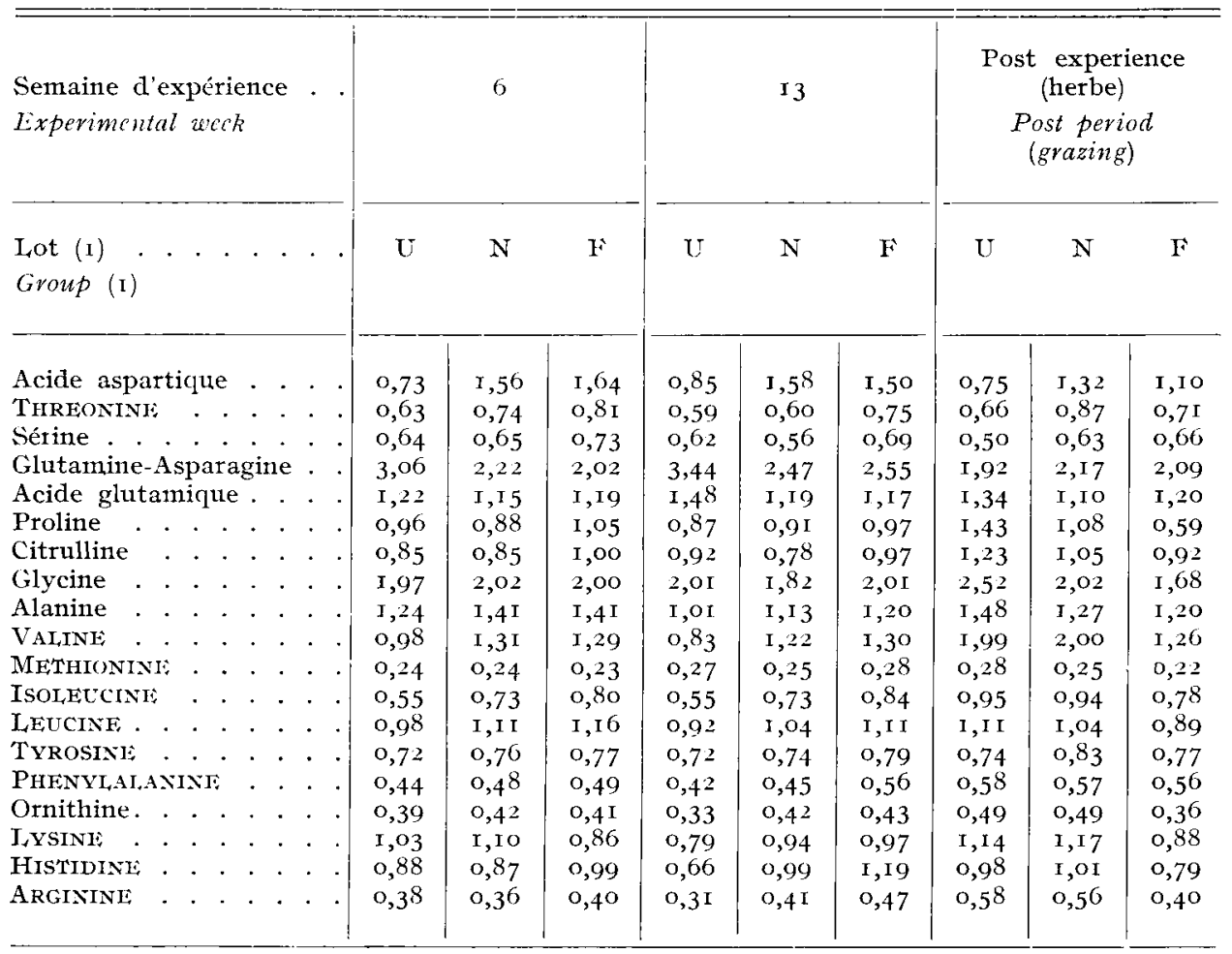
(I) IT : maïs-urée;
$\mathrm{N}:$ tourteau non traité; (r: maizc-urea;
$\mathbf{N}$ : untreated meal;
F : tourteau formolé.
F : treated meal.

\section{Influence du stade de lactation}

\section{- Expérience $I$.}

On observe entre la $I^{\text {re }}$ et $1 \mathrm{a} 5^{\mathbf{e}}$ semaine de lactation une diminution des teneurs en leucine, isoleucine, 1ysine, histidine et glycine et une augmentation des concentrations en acide aspartique, tyrosine et glutamine (+ asparagine). L'évolution 
observée en ce qui concerne l'histidine, la tyrosine et la glutamine ( + asparagine) entre la première et la $5^{\circ}$ semaine se poursuit jusqu'à la ro semaine de lactation.

\section{- Expérience $I I$.}

L'évolution des teneurs entre la $2^{\mathrm{e}}$ et la $5^{\mathrm{e}}$ semaine de lactation dépend du lot d'animaux considérés.

Dans le cas de la ration carencée en azote, entre la $2^{\mathrm{e}}$ et $1 \mathrm{a} 5^{\mathrm{e}}$ semaine de lactation, les concentrations en acides aminés indispensables n'ont pratiquement pas varié alors que celles en acides aminés non indispensables ont augmenté d'environ 20 p. cent sauf en ce qui concerne la glycine et 1'alanine dont les concentrations sont pratiquement restées inchangées.

Chez les animaux recevant les tourteaux non traités, les différences de teneur sont généralement faibles. Des diminutions notables ne sont observées que pour l'acide glutamique, la glycine, la valine et la glutamine ( + - asparagine).

Les teneurs en la plupart des acides aminés diminuent dans le sang des vaches alimentées avec les tourteaux formolés. Les concentrations en certains acides aminés non indispensables (acides aspartique et glutamique et citrulline) sont cependant plus élevées en $5^{\mathrm{e}} \mathrm{qu}$ 'en $2^{\mathrm{e}}$ semaine de lactation.

\section{- Expérience III.}

Pour un grand nombre d'acides aminés, les valeurs observées au cours de l'expérience III (milieu de lactation) sont voisines de celles observées en début de lactation (expérience I et II). Cependant, c'est dans les échantillons prélevés au cours de l'expérience III que les teneurs en glycine et sérine sont les plus faibles et que celles en acide aspartique et glutamine (+ asparagine) sont les plus élevées.

\section{Influence d'une sous-alimentation azotée en début de lactation (expérience II)}

Les teneurs de la plupart des acides aminés libres et en particulier des acides aminés indispensables (sauf méthionine et phétnylalanine) sont diminuées par la carence en azote. C'est en $2^{\mathrm{e}}$ semaine de lactation que le phénomène est le plus accentué.

\section{Infuence du remplacement des tourteaux par de l'urée (expérience III)}

L'incorporation d'urée au concentré en remplacement des tourteaux a eu pour conséquence un abaissement des teneurs en acide aspartique, ell acides aminés à chaînes ramifiées et, dans le prélèvement effectué au cours de la $13^{\mathrm{e}}$ semaine, en lysine et en histidine. En revanche, les concentrations de 1'acide glutamique et de la glutamine ( + asparagine) ont été augmentées.

\section{Influence de la mise à l'herbe}

\section{- Expérience II.}

A la suite de la mise à $1^{\prime}$ herbe $\left(5^{\mathrm{e}}\right.$ mois de lactation), on constate une augmentation dans le sang des vaches de la teneur de la plupart des acides aminés indispensables, en particulier en valine, méthionine, isoleucine et lysine par rapport 
au prélèvement réalisé en $5^{\mathrm{e}}$ semaine de lactation. Ces augmentations sont surtout sensibles chez les animaux soumis à une restriction azotée en début de lactation. Les concentrations en acides aminés non indispensables sont peu modifiées, mais parfois diminuées (acide aspartique, proline, glycine).

\section{- Expórience $I I I$.}

Le pâturage n'a provoqué une augmentation des teneurs en acides aminés libres du sang et en particulier en certains acides aminés indispensables (valine, isoleucine, 1ysine et phénylalanine) que chez les animaux ayant reçu précédemment la ration comportant de l'urée et à un degré moindre chez ceux ayant consommé les tourteaux non traités. En revanche, des diminutions notables sont observées chez les vaches qui avaient reçu les tourteaux formolés durant l'expérience. Pour les animaux des 3 lots, on note un abaissement des teneurs en acide aspartique et en glutamine ( + asparagine).

\section{Discussion}

Les teneurs en acides libres du sang des animaux sont en général plus faibles, surtout en ce qui concerne la méthionine et la phénylalanine que celles observées au cours d'une expérience antérieure (CHAMPREDON, PIOx et VÉRITÉ, I974) réalisée avec des vaches laitières recevant des rations à base d'ensilage de maìs. Cette constatation permet de supposer que la fourniture d'acides aminés et en particulier de méthionine aux tissus utilisateurs n'est pas excédentaire puisque 1'aminoacidémie reflète l'équilibre existant entre les apports et les utilisations. Le traitement des tourteaux au formol s'est traduit généralement par une augmentation des teneurs sanguines en valine, isoleucine, leucine, histidine et proline et par une diminution des concentrations en glutamine ( + asparagine) et dans un nombre limité de cas en lysine. Les différences les plus marquées correspondent aux périodes durant lesquelles les quantités de tourteaux distribuées aux animaux étaient les plus élevées. Une nette augmentation des tencurs sanguines en acides aminés à chaînes ramifiées et en particulier en valine à la suite du traitement de tourteaux ou de protéines a déjà été observée par ailleurs (FAICHNEy, I974; BRODERICK, SATTER et HARPER, I974). Elle peut se comparer à celle que l'on constate à la suite d'une élévation dut taux azoté de la ration qui a des conséquences similaires à l'infusion de protéines par voie post-ruminale (BRODERICK, KOWAI,CZYK et SatTer, i970; Derrig, Clark et IAvis, I974).

Cette augmentation est observée bien que les acides aminés à chaînes ramifiées soient prélevés dans le sang par la glande mammaire en quantités très supérieures à celles qui sont incorporées dans les protéines du lait (BICKERSTAFFE, Annison et Linzeli, I974; Spires et al., I975), et que la formolation des tourteaux ait entraîné une élévation de la production laitière des vaches. Ces composés ne sont donc pas des facteurs limitants primaires et leur utilisation pour la synthèse des matières azotées de lait est sans doute réduite par la fourniture en quantités insuffisantes de certaines autres substances (méthionine, phénylalanine, 1ysine) par le tube digestif.

L'élévation des teneurs sanguines en histidine et en proline chez les vaches consommant les tourteaux formolés est due au fait que les tourteaux de soja et de colza sont plus riches en ces 2 composés que les protéines microbiennes. I a dimi- 
nution des concentrations en lysine du sang, sans doute sous-estimée par suite de la formation de N-méthyl-lysine (REIs et Tuxks, I973; FAIChNEy, I974) sous l'action du formol, peut s'expliquer par le fait que les protéines des tourteaux utilisés en renferment moins que les protéines microbiennes.

La tendance à l'augmentation des teneurs sanguines en certaines acides aminés non indispensables au cours de la lactation, traduit une diminution de la néoglucogénèse à partir de ces composés due à une amélioration du bilan énergétique des animaux. La concentration relativement élevée en glycine observée en début de lactation est sans doute le résultat d'un important catabolisme protéique lié entre autre à l'involution de l'utérus qui se produit immédiatement après la parturition (CHAMPREDON et PION, I972).

I,es concentrations en acides aminés indispensables (sauf thréonine et lysine) en $5^{\mathrm{e}}$ semaine de lactation (expérience II) chez les vaches sous alimentées en azote sont très proches de celles observées en $6^{\mathrm{e}}$ semaine de l'expérience III dans le cas des animaux consommant l'aliment concentré comportant de l'urée et traduisent une fourniture réduite aux tissus des animaux. Cette similitude indique que les différences entre les apports d'acides aminés indispensables à 1'organisme et les utilisations pour la production laitière sont voisines. Dans un cas (distribution d'urée), une partie de l'azote alimentaire est mal utilisée car l'ammoniogénèse importante qui a lieu dans le rumen aboutit à un gaspillage notable de l'azote uréique qui franchit la paroi du rumen sous forme d'ammoniac et n'est pas utilisée pour la protéosynthèse bactérienne; la présence dans le sang de quantités importantes de glutamine ( + asparagine) reflète bien ce phénomène. Dans l'autre cas (sous alimentation azotée) le recyclage de l'urée sanguine dans le rumen permet une bonne utilisation de l'azote alimentaire apporté en quantité limitée.

La mise à l'herbe des vaches laitières à un stade où leurs besoins azotés et énergétiques sont diminués du fait de la décroissance de la production laitière, se traduit généralement par une augmentation des teneurs sanguines en acides aminés indispensables, mettant en évidence une élévation des apports d'acides aminés par rapport aux besoins des tissus des animaux. Cela n'est cependant pas le cas en ce qui concerne les vaches ayant consommé les tourteaux formolés en milieu de lactation (expérience III), ce qui pourrait traduire aussi bien une consommation alimentaire trop faible qu'une augmentation du catabolisme des acides aminés induite par des apports excessifs durant la période précédente.

\section{Conclusion}

La distribution aux vaches laitières de tourteaux formolés a eu pour principale conséquence un apport complémentaire d'acides aminés et en particulier d'acides aminés indispensables aux tissus des animaux. Cette fourniture supplémentaire a permis une amélioration de la production laitière. Si les quantités d'acides aminés à chaînes ramifiées, d'histidine et parfois de thréonine, fournies en supplément grâce au traitement des tourteaux par le formol ont été excédentaires il n'en a pas été de même pour les acides aminés à noyau aromatique, pour la lysine et surtout pour la méthionine. On peut donc supposer que si ces derniers composés et en particulier la méthionine avaient été apportés en quantités plus importantes dans l'intestin des animaux, leurs productions laitières auraient été améliorées de manière plus importante que dans le cas de cette série d'expériences. En outre, 
les utilisations des acides aminés à chaînes ramifiées, de l'histidine et de la thréonine auraient pu être notablement améliorées. Pour rendre le mélange absorbé dans l'intestin plus apte à la satisfaction des besoins en acides aminés indispensables des vaches laitières, il faut distribuer aux animaux sous forme protégée des protéines dont la composition soit complémentaire de celle des microbes et des matières azotées alimentaires non dégradées dans le rumen. Le choix desprotéines utilisées devra être guidé par la nécessité de corriger certaines carences souvent observées, en particulier en ce qui concerne la méthionine, l'histidine, la phénylalanine et les acides aminés à chaînes ramifiées.

Accepté pour publication en juin 1977.

\title{
Summary \\ Feeding formaldehyde treated meal to dairy cows \\ III. - The blood level of free amino acids as affected by treatment of meal, nitrogen source and nitrogen level of diet
}

\begin{abstract}
Dairy cows were fed isonitrogenous and isoenergetic diets containing maize silage and concentrate either during early lactation (experiment I and II) or during mid-1actation (experiment III). In each experiment, one group of cows received formaldehyde treated soybean and rapeseed meal and another group untreated meals. A third group received either a low nitrogen diet (experiment II) or a maize-urea mixture instead of soybean and rapeseed (experiment III). At the end of the experimental periods, the cows were fed meadow grass (VERITE and JOURNET, 1977).

The substitution of formaldehyde treated meal for untreated meal increased the blood levels of free valine, isoleucine, leucine, histidine and proline and decreased the blood levels of glutamine ( + asparagine) and lysine in some samples only (tables I, 2 and 3 ). The blood levels of free phenylalanine and mainly methionine were always low during the experimental period. The differences resulting from treatment of meal are explained by the increase in the amounts of most essential amino acids disappearing from the intestine (VÉRITE and al., I977) and the improvement of the milk production of the cows (VHRITÉ and JOURNET, I977). The utilization of histidine and branched chain amino acids by the mammary gland with the diets containing the treated meals seemed to be reduced by the amount of methionine, phenylalanine and lysine supplied to the animal tissues.

Feeding a low nitrogen diet as well as the replacement of meals by urea decreased most essential amino acid concentrations. These results suggest that methionine and phenylalanine are the first limiting amino acids for dairy cows fed maize silage.
\end{abstract}

\section{Références bibliographiques}

BICKERSTAFFث R., ANNISON E. F., LINZELL J. L., I974. The metabolism of glucose, acetate, lipids and amino acids in lactating dairy cows. J. agric. Sci. Camb., 82, 7 I-85.

Broderick G. A., Kowalczyk T., SATTER I. D., r97o. Milk production response to supplementation with encapsulated methionine per os or casein per abomasum. J. Dairy Sci,, 53 , I 7 I 4 -I $72 \mathrm{I}$.

Broderick G. A., SATTER L. D., HARPER A. E., I974. Use of plasma amino acid concentration. to identify limiting amino acids for milk ptoduction. J. Dairy Sci, 57, IOI5-1023.

Champredon C., Pion R., ig72. Evolution de l'aminoacidémie libre de chèvres au début de la lactation. C. R. Soc. Biol., 166, 378-38 I.

Champreidon C., PION R., VERITE R., I974. Influence de quelques facteurs alimentailes sur l'aminoacidémie libre de vaches laitières recevant des rations à base d'ensilage de maïs. $A n n$. Biol. anim. Bioch. Biophys., 14, 813-819. 
DHRrig R. G., Clark J. H., DAvis C. I., I974. Effect of abomasal infusion of sodium caseinate on milk yield, nitrogen utilization and amino acid nutrition of the dairy cow. $J . N u t r ., 104$, I 5 I-I 59.

Faiciney G. J., 1974. Effects of formaldehyde treatment of casein and peanut nieal sufplements on amino acids in digesta and plasma of lambs and sheep. Aust. J. Agric. Res., 25, 583-598.

REIS P. J., TUNks D. A., I973. Influence of formaldehyde treated casein supplements on the concentration of epsilon - N - methyllysine in sheep plasma. Aust. J. Biol. Sci., 26, I I27-I I36.

SPIRLS H. R., ClaRK J. H., DERRIG R. G., DAVIS C. L., I975. Milk production and nitrogen utilisation in response to postruminal infusion of sodium caseinate in lactating cows. $J$. Nutr., 105, I I I I-I I 2 I.

VFritis R., PONCFi C., Cham S., PION R., 1977. Utilisation des tourteaux traités au formol par les vaches laitières. I - Aspects digestifs. Ann. Zootech., 26, I67-I8I.

VERITE: R., JouRNFT M., J 977 . Utilisation des tourteaux traités au formol par les vaches laitières. II - Effects sur la production laitière du traitement des tourteaux et du niveau d'apport azoté au début de la lactation. Ann. Zootech., 26, I $83-205$. 